\title{
Transcription factor ZFP38 is essential for meiosis prophase I in male mice
}

\author{
Zechen Yan¹, Dandan Fan², Qingjun Meng ${ }^{1}$, Jinjian Yang ${ }^{1}$, Wei Zhao ${ }^{1}$, Fei Guo, \\ Dongjian Song ${ }^{1}$, Ruiming Guo ${ }^{1}$, Ke Sun $^{1}$ and Jiaxiang Wang ${ }^{1}$ \\ ${ }^{1}$ Department of Surgery, The First Affiliated Hospital of Zhengzhou University, Zhengzhou, \\ Henan, China and ${ }^{2}$ Henan Academy of Medical and Pharmaceutical Science, Zhengzhou, Henan, China \\ Correspondence should be addressed to J Wang; Email: wangjx72@yahoo.com
}

\begin{abstract}
The production of haploid gametes by meiosis is a cornerstone of sexual reproduction and maintenance of genome integrity. Zfp38 mRNA is expressed in spermatocytes, indicating that transcription factor ZFP38 has the potential to regulate transcription during meiosis. In this study, we generated Zfp38 conditional knockout mice (Zfp3 $8^{\text {flox/flox }}$, Stra8-Cre, hereafter called Zfp38 cKO) and found that spermatogenesis did not progress beyond meiosis prophase I in Zfp38 cKO mice. Using a chromosomal spread technique, we observed that Zfp38 cKO spermatocytes exhibited a failure in chromosomal synapsis observed by SYCP1/SYCP3 double staining. Progression of DNA double-strand breaks (DSB) repair is disrupted in Zfp38 cKO spermatocytes, as revealed by $\gamma$-H2AX, RAD51 and MLH1 staining. Furthermore, the mRNA and protein levels of DSB repair enzymes and factors that guide their loading onto sites of DSBs, such as RAD51, DMC1, RAD51, TEX15 and PALB2, were significantly reduced in Zfp38 cKO spermatocytes. Taken together, our data suggest that ZFP38 is critical for the chromosomal synapsis and DSB repairs partially via its regulation of DSB repairassociated protein expression during meiotic progression in mouse.

Reproduction (2016) 152 431-437
\end{abstract}

\section{Introduction}

Spermatogenesis is a highly complex cell differentiation process necessary for continued renewal of the spermatozoa population. Central to this unique process is meiosis, which is responsible for the production of haploid spermatozoa as well as for the maintenance of genome integrity (Chen \& Liu 2015). Meiosis errors and genetic disruptions can cause aneuploidy and infertility (Matzuk \& Lamb 2008). Prophase I of meiosis I is initiated in the primary spermatocytes and this stage can be divided into the leptotene, zygotene, pachytene, diplotene and diakinesis substages (Petronczki et al. 2003). During these substages of prophase I, the chromosomes undergo a number of changes that allow for the pairing of homologs and the exchange of genetic information between nonsister chromatids. Homologous chromosomes are connected by the synaptonemal complex (SC), which consists of the central element, axial/lateral elements and transverse filaments (de Boer \& Heyting 2006, Costa \& Cooke 2007). Homologous recombination involves the introduction of double-strand breaks (DSBs) into the genome by type II-like topoisomerase SPO11 complex (Handel 2004, Lam \& Keeney 2015, Robert et al. 2016, Vrielynck et al. 2016). DSBs are subsequently repaired by the loading of RAD51 and DMC1 recombinases (Pittman et al. 1998). TEX15, BRCA1, BRCA2 and PALB2 have been suggested to mediate RAD51 and DMC1 loading onto sites of DSBs (Scully et al. 1997, Davies et al. 2001, Yang et al. 2008, Simhadri et al. 2014).

A previous study suggests that the Zfp38 transcript can be detected at a highly elevated level during spermatogenesis (Chowdhury et al. 1992). Its expression accompanies the progression from pachytene spermatocytes to round spermatids (Chowdhury et al. 1992), suggesting that ZFP38 has the potential to regulate transcription during meiosis and/or spermiogenesis. However, the role of ZFP38 in vivo is still undiscovered.

In this study, Zfp38 conditional knockout mice (Zfp38 floxflox, Stra8-Cre; hereafter called Zfp38 cKO) were used to study the physiological roles of ZFP38 in spermatogenesis. We found that Zfp38 cKO male mice were completely infertile, due to meiotic arrest. Chromosomal synapsis and DSB repairs were impaired in Zfp38 cKO spermatocytes. Furthermore, ZFP38 deletion caused significantly reduced expression of RAD51, DMC1, TEX15, BRCA1/2 and PALB2. Our study suggests that ZFP38 is a novel spermatocyte factor required for the progression of meiotic prophase I in male mice. 


\section{Materials and methods}

\section{Generation of Zfp38 cKO mice}

All procedures and care of animals were performed in accordance with the Animal Care and Use Committee (ACUC) of The First Affiliated Hospital of Zhengzhou University. This study (protocol \#140312) was specifically approved by ACUC of The First Affiliated Hospital of Zhengzhou University. All mice were housed under controlled photoperiod conditions (lights on 0800-2000h) and supplied with food and $d_{d d H_{2}} \mathrm{O}$ ad libitum.

A targeting vector in which the Zfp38 exon 4 was flanked by a loxP site and a loxP-Neo cassette was constructed and introduced into mouse embryonic stem cells by electroporation. Following G418 selection, surviving colonies were screened for homologous recombinants by PCR. Clones were then injected into C57BL/6 (B6) blastocysts (Kaartinen \& Nagy 2001). Resultant male chimeras were identified by coat color and mated with wild-type females, resulting in successful germline transmission of the Zfp38 flox allele. Primers for genotyping the Zfp38 flox allele were as follows: P1, 5'-AGAGAGCAACACTGGGTCTAGTG; P2, 5'-GTGTTGTGACTGTATGAGTCAG; P3, 5'-TGTCCATG GGGAGATACAAGAC; P4, 5'-CTGTCTTTGAGCCATTTCTGCT. Zfp38 $8^{+/ f l o x}$ mice were mated with mice carrying the Stra8Cre transgene mice (obtained from The Jackson Laboratory, Stock No: 008208). DNA isolated from tail biopsies was used for genotyping. Genotyping for the Stra8-Cre transgene was performed as described previously (Sadate-Ngatchou et al. 2008).

\section{Tissue collection and staining}

The 4- or 8-week-old heterozygote (Zfp38 $8^{\text {flox/+ }}$, Stra8-Cre) and Zfp38 cKO (Zfp38 flox/flox, Stra8-Cre) mice were killed, and their testes were fixed in $4 \%$ paraformaldehyde (PFA) for H\&E and immunofluorescence staining. Briefly, issue sections were deparaffinized and rehydrated followed by antigen retrieval in $10 \mathrm{mM}$ sodium citrate buffer. Then, sections were blocked with goat serum in $0.3 \%$ Triton X-100 and incubated with anti-ZFP38 antibody (Abcam, ab140513, 1:200) or anti-MVH antibody (Abcam, ab13840, $1: 400$ ) or anti-SYCP3 (Abcam, ab15093, 1:400) or anti- $\gamma$ $\mathrm{H} 2 \mathrm{AX}$ (Abcam, ab2893, $1: 200$ ) at $4{ }^{\circ} \mathrm{C}$ overnight followed by incubation with the FITC- or TRITC-labeled secondary antibody for $1 \mathrm{~h}$. Negative control testis sections were incubated with secondary antibody in the absence of the primary antibody. The sections were counterstained with DAPI or PI (1:1000) to identify the nuclei.

\section{Spermatocyte cell isolation for expression analysis}

For isolation of spermatocytes, testes from 3-week-old mice were used. Spermatocyte cells were isolated as described previously (Baleato et al. 2005). Briefly, testes were isolated, decapsulated and incubated for $15 \mathrm{~min}$ each in $0.5 \mathrm{mg} / \mathrm{mL}$ collagenase/DMEM with agitation and then in $0.25 \%$ trypsin/EDTA in DMEM. The tubules were dissociated manually by pipetting and washed in $0.5 \%$ BSA in DMEM by centrifugation. Cell pellets were resuspended in DMEM and filtered twice through a $70 \mu \mathrm{M}$ membrane, then separated over a BSA gradient. The BSA gradient was prepared as described previously (Bellve et al. 1977).

\section{Protein extraction and Western blot analysis}

Tissue and spermatocytes were lysed in a radioimmunoprecipitation assay lysis buffer containing protease inhibitor cocktail tablets (Roche). Protein concentrations were measured using the Bradford assay. The proteins were electrophoresed under reducing conditions on $10 \%$ SDS-PAGE gels and transferred to nitrocellulose membranes. The blots were incubated with anti-ZFP38 antibody (Abcam, ab140513, 1:1000) or anti-RAD51 (Abcam, ab88573, 1:1000) or anti-DMC1 (Abcam, ab96613, 1:1000) or anti-TEX15 (Abcam, ab122364, 1:1000) or anti-PALB2 (Abcam, ab202970, 1:1000) or anti-PLZF (Santa Cruz, sc28319, 1:500) or anti-SYCP3 (Abcam, ab97672, 1:1000) or anti-WT1 (Abcam, ab89901, 1:1000) or anti-P450 Scc (Boster, Wuhan, China, BA3699, 1:1000) overnight at $4{ }^{\circ} \mathrm{C}$ followed by Dye $800 \mathrm{CW}$ secondary antibody for $1 \mathrm{~h}$ at room temperature. The protein level was normalized and plotted against $\beta$-tubulin (Cell Signaling Technology, \#2146, 1:2000). Signals were detected using the Western blotting Detection System (Odyssey, MA, USA).

\section{Chromosome spreads}

Chromosome spreads were prepared by the dry-down technique as described previously (Holloway et al. 2011, Chen et al. 2016). Briefly, testis tissues were dissected, and tubules were dissociated with a pipet in PBS. Cells were ruptured by adding an equal amount of hypotonic buffer $(30 \mathrm{mM}$ Tris- $\mathrm{HCl}$ $\mathrm{pH}$ 8.2, $50 \mathrm{mM}$ sucrose, $17 \mathrm{mM}$ sodium citrate, $5 \mathrm{mM}$ EDTA, $0.5 \mathrm{mM}$ DTT and protease inhibitors) and then mixing the solution 1:2 with $100 \mathrm{mM}$ sucrose just before spreading on slides that were preincubated with $1 \%$ PFA. The slides were then dried for $1 \mathrm{~h}$. Immunostaining of spermatocyte spreads was performed similar to the immunofluorescence analysis (as described above). The following antibodies were used: anti-SYCP1 (Abcam, ab15087, 1:400), anti-SYCP3 (Abcam, ab97672, 1:400), anti- $\boldsymbol{\gamma}-\mathrm{H} 2 \mathrm{AX}$ (Abcam, ab2893, 1:200), anti-RAD51 (Millipore, ABE257, 1:200) and anti-MLH1 (Abcam, ab92312, 1:200).

\section{Gene expression analysis by quantitative real-time PCR}

Tissue and spermatocyte RNA was extracted using TRIzol (Invitrogen) according to the manufacturer's protocol. RNA samples were subjected to reverse transcription using a PrimeScript RT Reagent Kit (Takara). The reactions were run in triplicate in three independent experiments. The cycle threshold $\left(C_{T}\right)$ values for the samples were normalized to the corresponding Gapdh $\mathrm{C}_{\mathrm{T}}$ value, and relative expression levels were calculated using the $\Delta \Delta \mathrm{C}_{\mathrm{T}}$ method. The primer sequences are provided in Supplementary Table 1, see section on supplementary data given at the end of this article. 


\section{Statistical analysis}

Experiments were repeated at least three times. Data were evaluated for significance using Student's $t$-test. The results are presented as the mean \pm S.E.M. $P<0.05$ was deemed significant.

\section{Results}

\section{ZFP38 expression in mouse testes}

The findings of a previous study suggested that Zfp38 mRNA is expressed from pachytene spermatocytes to round spermatids (Chowdhury et al. 1992). In this study, we observed that ZFP38 protein was predominantly expressed in testes (Fig. 1A). Only a small amount of ZFP38 expression was observed in ovary and kidney. Furthermore, ZFP38 expression in testis started from postnatal day 7 (PND7) and did not differ at PND 14, 21 and 28 (Fig. 1B). To evaluate the ZFP38 expression in different testicular

A

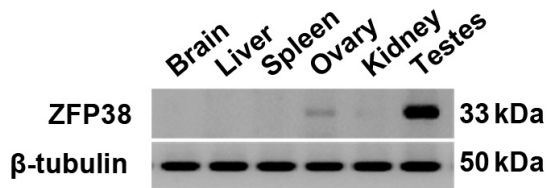

B

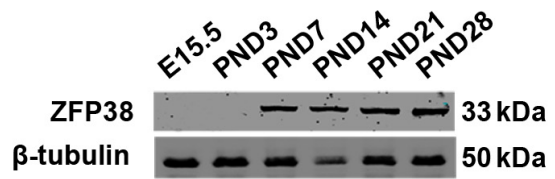

C

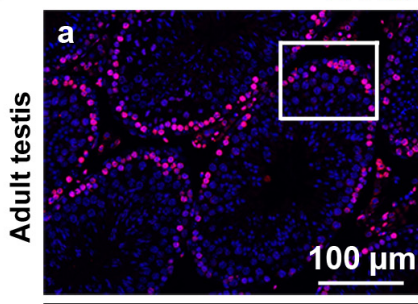

ZFP38/DAPI
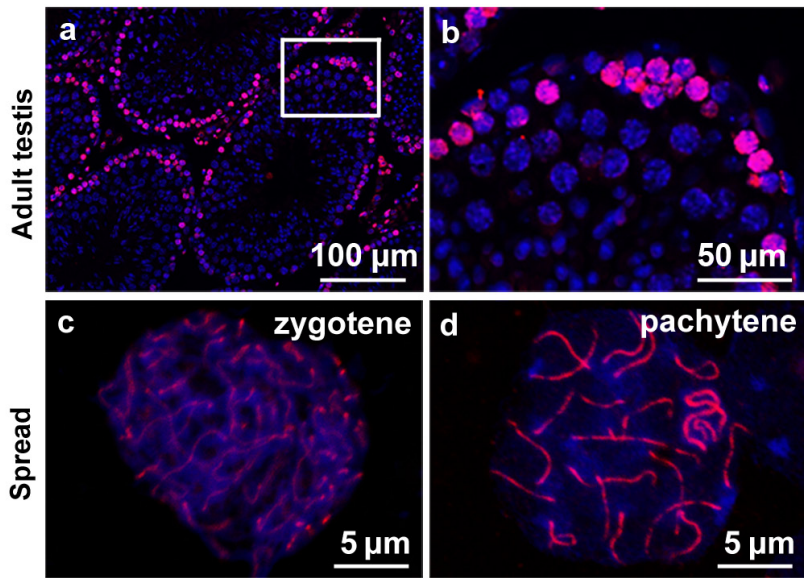

pachytene

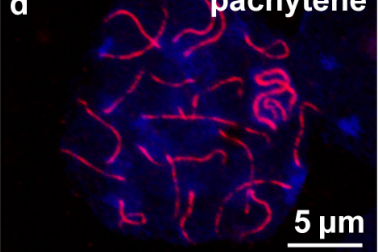

Figure 1 Expression of ZFP38 protein in mouse testes. (A) Expression of ZFP38 in mouse tissues, including brain, liver, spleen, ovary, kidney and testes. (B) Expression of ZFP38 in testes at different developmental stages, including E15.5, PND 3, 7, 14, 21 and 28. $\beta$-tubulin served as a protein-loading control in Western blot analysis. (C) The cellular localization of ZFP38 (red) in sections of adult testes was displayed by immunofluorescent staining. The nuclei are counterstained with DAPI (blue). Representative chromosome spreads of zygonema and pachynema are labeled with anti-ZFP38 (red) antibodies. Scale bar in a, $100 \mu \mathrm{m}$; in b, $50 \mu \mathrm{m}$; in c and d, $5 \mu \mathrm{m}$. cells, we performed immunofluorescence staining and observed that ZFP38 was specifically present in spermatocytes (Fig. 1C; a and b). ZFP38 was detected predominantly in chromosome axes of meiocytes and its levels increased from zygonema to pachynema (Fig. 1C; c and d).

\section{Specific disruption of Zfp38 in meiotic spermatocytes}

To assess the in vivo function of ZFP38 during meiosis, we generated spermatocyte-specific Zfp38 gene knockout (Zfp38 flox/flox, Stra8-Cre) mice. LoxP sites flank exon 4 of the Zfp38 allele, and recombination of the loxp sites using Stra8-Cre recombinase resulted in the removal of exon 4 (Fig. 2A). The Stra8-Cre transgene is expressed in preleptotenestage spermatocytes (Sadate-Ngatchou et al. 2008). The successful acquisition of Zfp38 cKO mice was determined by polymerase chain reaction (PCR) amplification (Fig. 2B). We confirmed that ZFP38 was successfully depleted in Zfp38 cKO testes using Western blot analysis (Fig. 2C). ZFP38 was absent in spermatocytes in Zfp38 cKO mice, as evidenced by immunofluorescence staining (Fig. 2D).

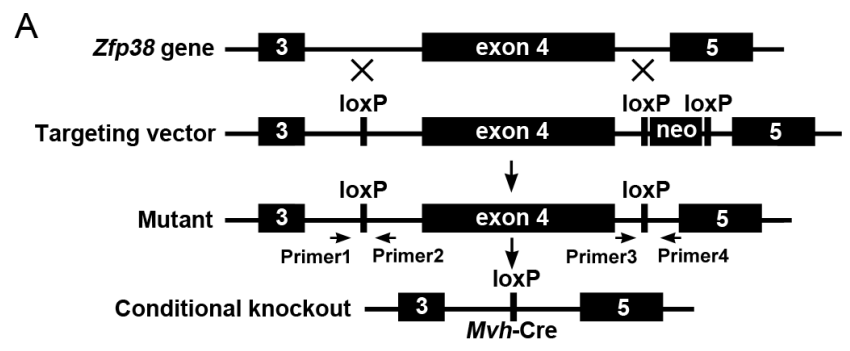

B

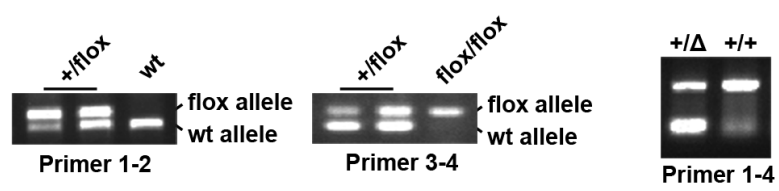

C

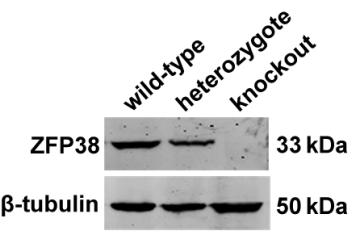

D

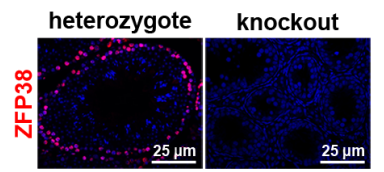

Figure 2 Generation of Zfp38 cKO mice. (A) Targeting strategy and the creation of $Z f p 38$ cKO mice. In the targeted allele, a loxP site and a pgk-Neo cassette flanked by two loxP sites were inserted into intron 4. (B) PCR analysis detected the presence of 5' (PCR: P1-P2) and 3' (PCR: P3-P4) loxP sites for genotyping the wild-type $(+/+)$, heterozygous (flox/+) and homozygous (flox/flox) mice, and examined the deletion of exon 4 in the Zfp38 $\Delta /+$ mice (PCR: P1-P4). (C) Knockout efficiency was confirmed by Western blotting in wild-type (line 1), heterozygote (line 2) and knockout (line 3) testes. The protein level was normalized against $\beta$-tubulin. (D) Staining analysis of ZFP38 (red) in heterozygote and knockout testes. Scale bars, $25 \mu \mathrm{m}$. 

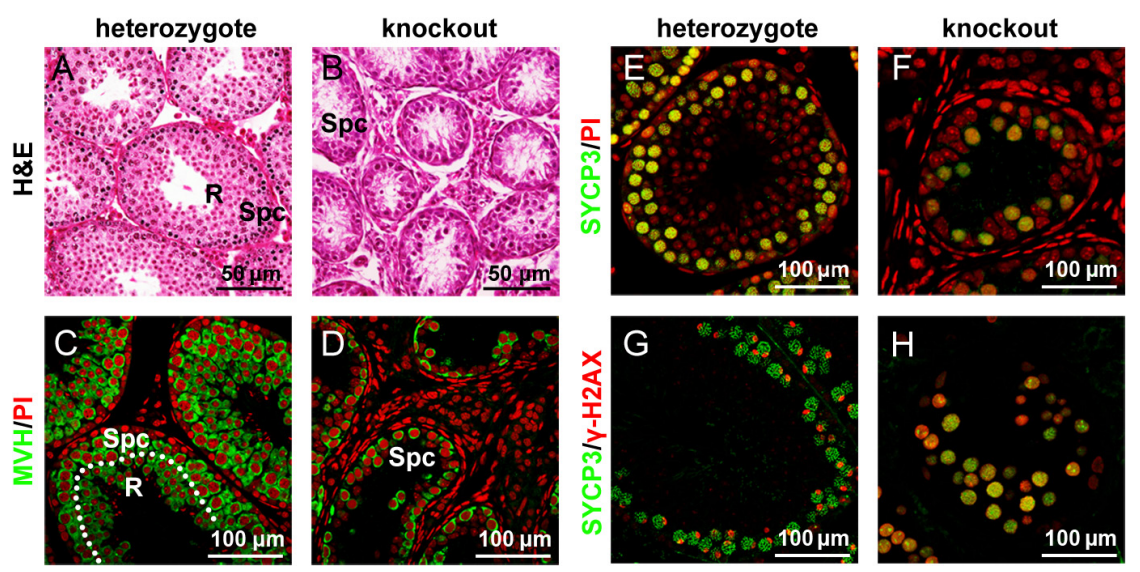

\section{Infertility and meiotic arrest in Zfp38 cKO mice}

Six- to eight-week-old Zfp38 cKO males failed to produce offspring after natural mating with wild-type C57 female mice (at a ratio of 1:2) for more than 4 months. In contrast, heterozygotes $\left(Z f p 38^{+/ f l o x}\right.$, Stra8-Cre) were fully fertile with an average litter size of $8.9 \pm 1.4$ (Supplementary Fig. 1A). Adult male Zfp38 cKO mice showed no apparent morphological defects in nonreproductive organs, and there was no difference in body weight between the Zfp38 cKO and heterozygote males (Supplementary Fig. 1B). However, testis weight of adult Zfp38 cKO mice was less than $40 \%$ of heterozygotes (Supplementary Fig. 1C), which was likely due to the loss of postmeiotic cells. Indeed, H\&E staining and MVH (a germ cell marker) immunofluorescence in 4-week-old heterozygote and Zfp38 cKO testes showed normal postmeiotic round spermatids in heterozygote testes (Fig. 3A and C), versus few cells of pachytene-like morphology and tubules lacking haploid cells in age-matched Zfp38 cKO testes (Fig. 3B and D). Furthermore, the meiosis in Zfp38 cKO spermatocytes was abnormal, as revealed by immunofluorescence staining of SYCP3 (a synaptonemal complex protein) and $\gamma-\mathrm{H} 2 \mathrm{AX}$ (a proxy for unrepaired breaks). Spermatocytes of heterozygotes exhibited a representative chromosomal expression pattern (woolball-like structure) (Fig. 3E), while no typical wool-balllike structure of chromosomes was observed in Zfp38 cKO spermatocytes (Fig. 3F). $\gamma-\mathrm{H} 2 \mathrm{AX}$ signals in heterozygotes were restricted to one spot (asynapsed $X Y$ body) after pachytene stage (Fig. 3G), whereas the same signal was dispersed in Zfp38 cKO spermatocytes (Fig. 3H).

\section{Failure of chromosomal synapsis and DSB repair in Zfp38 cKO spermatocytes}

To determine the cause of meiotic arrest and to gain mechanistic insight into this defect in Zfp38 cKO spermatocytes, we examined the assembly of the synaptonemal complex and DSB repair by surface spread analysis of spermatocytes. Using SYCP1/ SYCP3 (from the central and axial/lateral elements

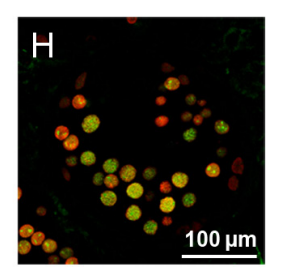

Figure 3 Meiotic arrest in Zfp38 cKO male mice. (A and B) $\mathrm{H} \& E$ staining of heterozygote and Zfp38 cKO testis section at 4 weeks. (C and D) Expression of MVH (a germ cell marker) in heterozygote and Zfp38 cKO testes. (E and F) Cell localization of SYCP3 in heterozygote and Zfp38 CKO testes. ( $\mathrm{G}$ and $\mathrm{H})$ Colocalization of SYCP3 and $\gamma-\mathrm{H} 2 \mathrm{AX}$ in heterozygote and Zfp38 cKO testes. The nuclei are counterstained with $\mathrm{PI}$ in $\mathrm{C}-\mathrm{F}$ (red). Spc, spermatocytes; R: round spermatids. Scale bars in A-B, $50 \mu \mathrm{m}$; in $\mathrm{C}-\mathrm{H}, 100 \mu \mathrm{m}$.

of synaptonemal complex) double staining of heterozygote pachytene spermatocytes, we observed perfect colocalization of SYCP1 and SYCP3 around

SYCP3
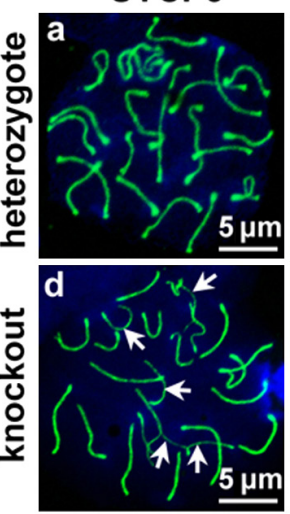

B
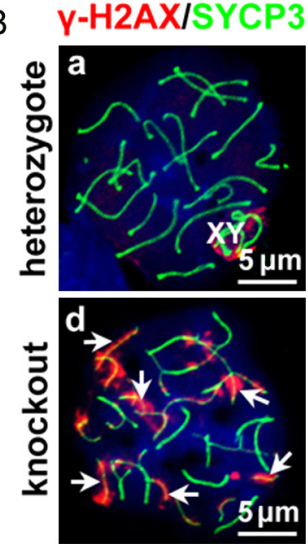

SYCP1
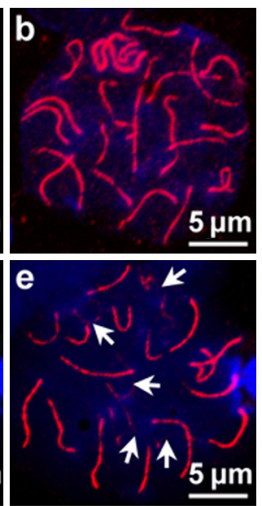

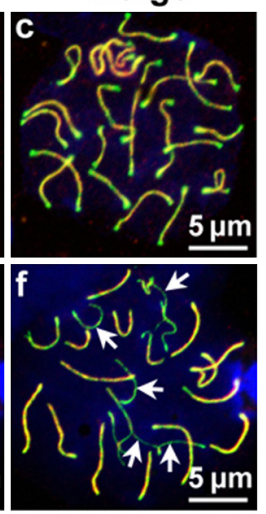

Merge

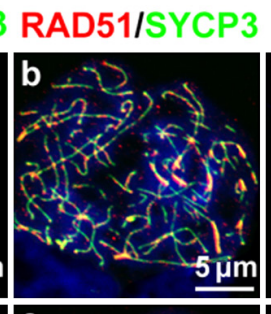

\section{MLH1/SYCP3}
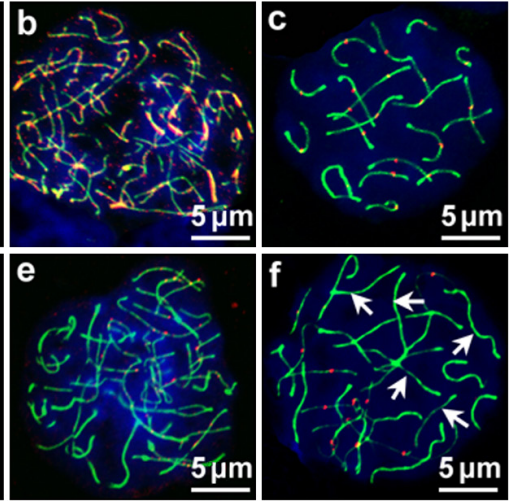

Figure 4 Aberrant chromosomal synapsis and failure of DSB repair in Zfp38 cKO spermatocytes. (A) Representative chromosome spreads of spermatocytes at PND 21 labeled with anti-SYCP3 (green) and anti-SYCP1 (red) antibodies. Arrows indicated asynapsed

chromosomes. The nuclei are counterstained with DAPI (blue). Scale bars, $5 \mu \mathrm{m}$. (B) Chromosome spreads from PND 21 heterozygote and Zfp38 cKO mice were stained with antibodies against $\gamma$-H2AX (a, d) or RAD51 (b, e) or MLH1 (c, f). The nuclei are counterstained with DAPI (blue). Arrows in d indicated residual expression of $\gamma-\mathrm{H} 2 \mathrm{AX}$ in autosomes, and arrows in $\mathrm{f}$ indicated autosomes completely lacked a MLH1 focus. Scale bars, $5 \mu \mathrm{m}$. 
the synaptonemal complex (Fig. 4A; a-c). However, obviously, unsynapsed or partially synapsed chromosomes (arrows) were observed in Zfp38 cKO spermatocytes (Fig. 4A; d-f). Both the number of spreads with partial synapsis ( $85 \pm 4$ for cKO vs $9 \pm 2$ for heterozygote; 100 cells counted from 3 males) and the average number of asynapsed chromosomes per nucleus ( $7 \pm 2$ for cKO vs $\sim 0$ for heterozygote; 100 cells counted from 3 males) were significantly increased in Zfp38 cKO pachynema. $\gamma-\mathrm{H} 2 \mathrm{AX}$ staining was undetectable on autosomes and restricted to the largely asynapsed $X Y$ body in heterozygote pachynema (Fig. 4B). Conversely, $\gamma-\mathrm{H} 2 \mathrm{AX}$ staining of Zfp38 CKO pachynema was sustained in asynapsed autosomal homologs (Fig. 4B; arrows). Furthermore, we detected the distribution of RAD51, which is a critical component of DNA repair complex. There was a dramatic decrease in the number of RAD51 foci $(160 \pm 22$ in heterozygote vs $43 \pm 17$ in cKO; 100 cells counted from 3 males) in Zfp38 cKO zygonema compared with the heterozygote zygonema (Fig. 4B; b and e). We also found a significant decrease in the number of MLH1 foci in Zfp38 cKO pachynema $(13 \pm 3$ for cKO vs $24 \pm 2$ for heterozygote; 100 cells counted from 3 males). In addition, $87.9 \%$ of Zfp38 cKO pachytene cells contained at least one autosome that completely lacked a MLH1 focus compared with $3.6 \%$ of heterozygote pachynema (100 cells counted from 3 males) (Fig. 4B; c and f).

A

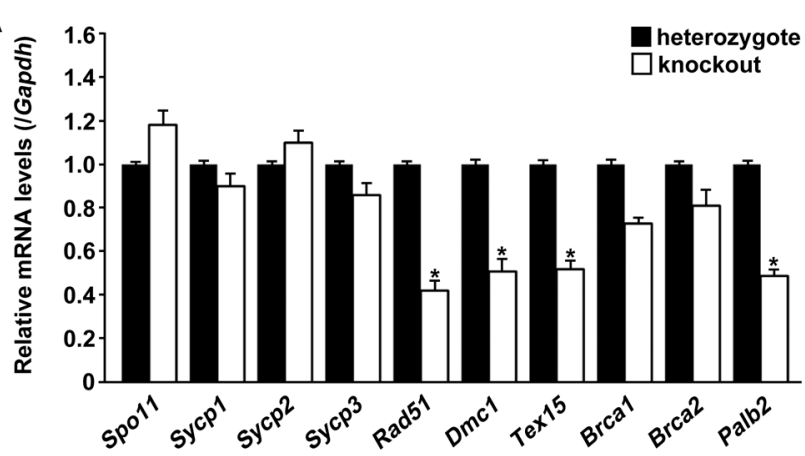

B
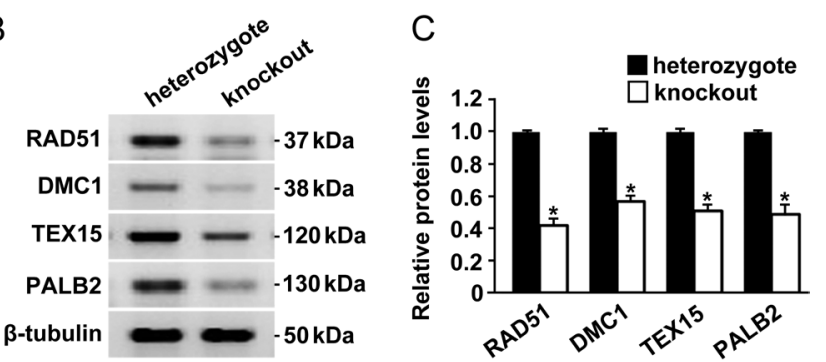

Figure 5 Attenuated expression of DSB repair-associated factors. (A) The mRNA levels of Spo11, Sycp1/3, Rad51, Tex15, Brca1/2 and Palb2 in heterozygote and Zfp38 cKO spermatocytes. Gapdh served as the internal control gene. (B) Protein levels of RAD51, DMC1, TEX15 and PALB2 in heterozygote and Zfp38 cKO spermatocytes. $\beta$-tubulin served as a protein-loading control. The data are expressed as the mean \pm S.E.M. ${ }^{*} P<0.05$.

\section{Downregulation of DSB repair-associated factors after ZFP38 deletion in spermatocytes}

To determine the underlying reasons of aberrant chromosomal synapsis and failure of DSB repair in Zfp38 cKO spermatocytes, we detected the expression of SPO11 (a DSB inducer), synaptonemal complex proteins (SYCP1-3) and DSB repair-associated factors (RAD51, DMC1, TEX15, BRCA1/2 and PALB2). We observed that the mRNA levels of Spo11, Sycp1, Sycp2, Sycp3, Brca 1 and Brca2 were not significantly altered between heterozygote and Zfp38 cKO spermatocytes. However, the mRNA levels of DSB repair-associated genes, such as Rad51,Dmc1, Tex15 and Palb2, were significantly reduced in Zfp38 cKO spermatocytes (Fig. 5A). Western blot analysis further indicated that the protein levels of RAD51, DMC1, TEX15 and PALB2 were significantly downregulated in Zfp38 cKO spermatocytes (Fig. 5B and C).

\section{Discussion}

Expression analysis of ZFP38 strongly suggests a role in meiotic progression in male germ cells. In the male germ line, ZFP38 protein emerged from PND7. Subsequently, the expression of ZFP38 appeared relatively constant. Antibody localization of ZFP38 confirmed its presence in zygotene and pachytene spermatocytes in testis sections. Although Zfp38 transcripts were expressed from pachytene spermatocytes to round spermatids (Chowdhury et al. 1992), ZFP38 protein was undetectable in round spermatids.

Conditional knockout strategy was used to investigate the role of transcription factor ZFP38 in mouse spermatogenesis. Zfp38 cKO male mice were completely infertile and exhibited meiotic arrest. Zfp38 cKO testes showed few spermatocytes, without round spermatids. The role for ZFP38 in meiosis was corroborated by its restricted expression in spermatocytes.

Our chromosome spread data indicated ZFP38 was required for spermatocytes to progress meiotic prophase I, as both recombination and DSB repair were affected in Zfp38 cKO mice. Two RecA homologs, RAD51 and its counterpart DMC1, mediate homologous chromatid invasion and promote homologous chromosome pairing and synapsis in spermatocytes (Pittman et al. 1998, Yoshida et al. 1998). TEX15 (Yang et al. 2008), BRCA1 (Scully et al. 1997), BRCA2 (Davies et al. 2001) and PALB2 (Simhadri et al. 2014) mediate loading of the RAD51 and DMC1 recombinases onto sites of DSBs. Deficiency in genes encoding synaptonemal complex proteins exhibits similar phenotypes - e.g., knockouts of Sycp1 (de Vries et al. 2005), Scyp2 (Yang et al. 2006), Sycp3 (Yuan et al. 2000), Syce 1 (Bolcun-Filas et al. 2009), Syce2 (Bolcun-Filas et al. 2007), Syce3 (Schramm et al. 2011) and Tex12 
(Hamer et al. 2008) produce male infertility with an arrest during prophase. Given that the deficiency of these genes exhibits similarities to the phenotype of Zfp38 cKO male mice, we detected the expression of genes encoding synaptonemal complex proteins and DSB repair-associated factors. The mRNA levels of Spo 11, Sycp 1, Sycp2, Sycp3, Brca 1 and Brca2 were normal, while the mRNA and protein levels of RAD51, DMC1, TEX15 and PALB2 were reduced in Zfp38 cKO spermatocytes. While the transcript abundance of Sycp 1-3 was not altered in Zfp38 gKO spermatocytes, SYCP1 and SYCP3 proteins do not colocalize properly in these cells. Whether transcription factor ZFP38 regulates Rad51, Dmc1, Tex15 and Palb2 directly or indirectly needs further investigation. Genome-wide transcriptome analysis (microarray or RNA-seq) will be helpful to discern the regulatory mechanisms of transcription factor ZFP38 in meiocytes.

We found that both MLH1 foci and RAD51 foci were reduced in Zfp38 cKO spermatocytes, which might be the consequence of the reduced expression of DSB repair-associated proteins. Although we observed that $\gamma-\mathrm{H} 2 \mathrm{AX}$ foci responded equally well and appeared at the damage sites during the zygotene stage in both the control and the Zfp38 cKO spermatocytes, with the same staining pattern and comparable intensity, we could not exclude the possibility that DSB formation was decreased in Zfp38 cKO spermatocytes. A recent study suggests that the presence of $\gamma-\mathrm{H} 2 \mathrm{AX}$ foci should not be considered an unequivocal marker of DSB (Rybak et al. 2016). Although the level of Spo11 mRNA was normal in Zfp38 cKO spermatocytes, other factors that are needed for DSB formation or forming complex with SPO11 (Robert et al. 2016, Vrielynck et al. 2016) may be limiting.

Meiotic sex chromosome inactivation (MSCl) enables the unpaired sex chromatin to bypass meiotic synapsis checkpoints and is essential for meiotic progression in males (Modzelewski et al. 2012). We found that ZFP38 staining was also evident in sex body at pachynema. Thus, we suggest that MSCI may be another possible reason of meiotic arrest in Zfp38 cKO mice and whether Zfp38 cKO spermatocytes fail to silence sex-linked transcripts and lead to apoptosis needs further investigation.

In summary, ZFP38 regulates chromosomal synapsis and DSB repair during meiotic prophase I. The meiotic arrest phenotype could be partially explained by the potential regulation of RAD51, DMC1, TEX15 and PALB2 by transcription factor ZFP38.

\section{Supplementary data}

This is linked to the online version of the paper at http://dx.doi. org/10.1530/REP-16-0225.

\section{Declaration of interest}

The authors declare that there is no conflict of interest that could be perceived as prejudicing the impartiality of the research reported.

\section{Funding}

This research was supported by the Medical Science and Technique Foundation of Henan Province (No. 201503055) and the Youth Innovation Fund of the First Affiliated Hospital of Zhengzhou University.

\section{Authors' contribution statement}

J W conceived and designed the experiments. Z Y, D F, Q M, J $Y$ and $W Z$ performed the experiments. F G, D S, R G and K S analyzed the data. J W wrote the paper.

\section{References}

Baleato RM, Aitken RJ \& Roman SD 2005 Vitamin A regulation of BMP4 expression in the male germ line. Developmental Biology 286 78-90. (doi:10.1016/j.ydbio.2005.07.009)

Bellve AR, Millette CF, Bhatnagar YM \& O'Brien DA 1977 Dissociation of the mouse testis and characterization of isolated spermatogenic cells. Journal of Histochemistry and Cytochemistry 25 490-494. (doi:10.1177/25.7.893996)

Bolcun-Filas E, Costa Y, Speed R, Taggart M, Benavente R, De Rooij DG \& Cooke HJ 2007 SYCE2 is required for synaptonemal complex assembly, double strand break repair \& homologous recombination. Journal of Cell Biology 176 741-747. (doi:10.1083/jcb.200610027)

Bolcun-Filas E, Hall E, Speed R, Taggart M, Grey C, de Massy B, Benavente R \& Cooke HJ 2009 Mutation of the mouse Syce1 gene disrupts synapsis and suggests a link between synaptonemal complex structural components and DNA repair. PLoS Genetics 5 e1000393. (doi:10.1371/journal.pgen.1000393)

Chen SR \& Liu YX 2015 Regulation of spermatogonial stem cell self-renewal and spermatocyte meiosis by Sertoli cell signaling. Reproduction 149 R159-R167. (doi:10.1530/REP-14-0481)

Chen SR, Hao XX, Zhang Y, Deng SL, Wang ZP, Wang YQ, Wang XX \& Liu YX 2016 Androgen receptor in Sertoli cells regulates DNA doublestrand break repair and chromosomal synapsis of spermatocytes partially through intercellular EGF-EGFR signaling. Oncotarget 7 18722-18735. (doi:10.18632/oncotarget.7916)

Chowdhury K, Goulding M, Walther C, Imai K \& Fickenscher H 1992 The ubiquitous transactivator Zfp-38 is upregulated during spermatogenesis with differential transcription. Mechanisms of Development 39 129-142. (doi:10.1016/0925-4773(92)90040-Q)

Costa Y \& Cooke HJ 2007 Dissecting the mammalian synaptonemal complex using targeted mutations. Chromosome Research 15 579-589. (doi:10.1007/s10577-007-1142-1)

Davies AA, Masson JY, Mcllwraith MJ, Stasiak AZ, Stasiak A, Venkitaraman AR \& West SC 2001 Role of BRCA2 in control of the RAD51 recombination and DNA repair protein. Molecular Cell 7 273-282. (doi:10.1016/S1097-2765(01)00175-7)

de Boer E \& Heyting C 2006 The diverse roles of transverse filaments of synaptonemal complexes in meiosis. Chromosoma 115 220-234. (doi:10.1007/s00412-006-0057-5)

de Vries FA, de Boer E, van den Bosch M, Baarends WM, Ooms M, Yuan L, Liu JG, van Zeeland AA, Heyting C \& Pastink A 2005 Mouse Sycp1 functions in synaptonemal complex assembly, meiotic recombination, and XY body formation. Genes \& Development 19 1376-1389. (doi:10.1101/gad.329705)

Hamer G, Wang H, Bolcun-Filas E, Cooke HJ, Benavente R \& Hoog C 2008 Progression of meiotic recombination requires structural maturation 
of the central element of the synaptonemal complex. Journal of Cell Science 121 2445-2451. (doi:10.1242/jcs.033233)

Handel MA 2004 The XY body: a specialized meiotic chromatin domain. Experimental Cell Research 296 57-63. (doi:10.1016/ j.yexcr.2004.03.008)

Holloway JK, Mohan S, Balmus G, Sun X, Modzelewski A, Borst PL, Freire R, Weiss RS \& Cohen PE 2011 Mammalian BTBD12 (SLX4) protects against genomic instability during mammalian spermatogenesis. PLoS Genetics 7 e1002094. (doi:10.1371/journal.pgen.1002094)

Kaartinen V \& Nagy A 2001 Removal of the floxed neo gene from a conditional knockout allele by the adenoviral Cre recombinase in vivo. Genesis 31 126-129. (doi:10.1002/gene.10015)

Lam I \& Keeney S 2015 Mechanism and regulation of meiotic recombination initiation. Cold Spring Harbor Perspectives in Biology 7 a016634. (doi:10.1101/cshperspect.a016634)

Matzuk MM \& Lamb DJ 2008 The biology of infertility: research advances and clinical challenges. Nature Medicine 14 1197-1213. (doi:10.1038/ nm.f.1895)

Modzelewski AJ, Holmes RJ, Hilz S, Grimson A \& Cohen PE 2012 AGO4 regulates entry into meiosis and influences silencing of sex chromosomes in the male mouse germline. Developmental Cell 23 251-264. (doi:10.1016/j.devcel.2012.07.003)

Petronczki M, Siomos MF \& Nasmyth K 2003 Un menage a quatre: the molecular biology of chromosome segregation in meiosis. Cell $\mathbf{1 1 2}$ 423-440. (doi:10.1016/S0092-8674(03)00083-7)

Pittman DL, Cobb J, Schimenti KJ, Wilson LA, Cooper DM, Brignull E, Handel MA \& Schimenti JC 1998 Meiotic prophase arrest with failure of chromosome synapsis in mice deficient for Dmc1, a germline-specific RecA homolog. Molecular Cell 1 697-705. (doi:10.1016/S10972765(00)80069-6)

Robert T, Nore A, Brun C, Maffre C, Crimi B, Bourbon HM \& de Massy B 2016 The TopoVIB-Like protein family is required for meiotic DNA double-strand break formation. Science 351 943-949. (doi:10.1126/ science.aad5309)

Rybak P, Hoang A, Bujnowicz L, Bernas T, Berniak K, Zar ABMA, Darzynkiewicz Z \& Dobrucki J 2016 Low level phosphorylation of histone $\mathrm{H} 2 \mathrm{AX}$ on serine 139 (gammaH2AX) is not associated with DNA double-strand breaks. Oncotarget 7 49574-49587. (doi:10.18632/oncotarget.10411)

Sadate-Ngatchou PI, Payne CJ, Dearth AT \& Braun RE 2008 Cre recombinase activity specific to postnatal, premeiotic male germ cells in transgenic mice. Genesis 46 738-742. (doi:10.1002/dvg.20437)
Schramm S, Fraune J, Naumann R, Hernandez-Hernandez A, Hoog C, Cooke HJ, Alsheimer M \& Benavente R 2011 A novel mouse synaptonemal complex protein is essential for loading of central element proteins, recombination, and fertility. PLoS Genetics 7 e1002088. (doi:10.1371/journal.pgen.1002088)

Scully R, Chen J, Plug A, Xiao Y, Weaver D, Feunteun J, Ashley T \& Livingston DM 1997 Association of BRCA1 with Rad51 in mitotic and meiotic cells. Cell 88 265-275. (doi:10.1016/S0092-8674(00)81847-4)

Simhadri S, Peterson S, Patel DS, Huo Y, Cai H, Bowman-Colin C, Miller S, Ludwig T, Ganesan S, Bhaumik M et al. 2014 Male fertility defect associated with disrupted BRCA1-PALB2 interaction in mice. Journal of Biological Chemistry 289 24617-24629. (doi:10.1074/jbc. M114.566141)

Vrielynck N, Chambon A, Vezon D, Pereira L, Chelysheva L, De Muyt A, Mezard C, Mayer C \& Grelon M 2016 A DNA topoisomerase VI-like complex initiates meiotic recombination. Science 351 939-943. (doi:10.1126/science.aad5196)

Yang F, De La Fuente R, Leu NA, Baumann C, McLaughlin KJ \& Wang PJ 2006 Mouse SYCP2 is required for synaptonemal complex assembly and chromosomal synapsis during male meiosis. Journal of Cell Biology 173 497-507. (doi:10.1083/jcb.200603063)

Yang F, Eckardt S, Leu NA, McLaughlin KJ \& Wang PJ 2008 Mouse TEX15 is essential for DNA double-strand break repair and chromosomal synapsis during male meiosis. Journal of Cell Biology 180 673-679. (doi:10.1083/ jcb.200709057)

Yoshida K, Kondoh G, Matsuda Y, Habu T, Nishimune Y \& Morita T 1998 The mouse RecA-like gene Dmc1 is required for homologous chromosome synapsis during meiosis. Molecular Cell 1 707-718. (doi:10.1016/S1097-2765(00)80070-2)

Yuan L, Liu JG, Zhao J, Brundell E, Daneholt B \& Hoog C 2000 The murine SCP3 gene is required for synaptonemal complex assembly, chromosome synapsis, and male fertility. Molecular Cell 5 73-83. (doi:10.1016/S1097-2765(00)80404-9)

Received 25 April 2016

First decision 16 May 2016

Revised manuscript received 18 July 2016

Accepted 4 August 2016 